Mathematical Modelling and Analysis

Volume 16 Number 2, June 2011, 199-219

Doi:10.3846/13926292.2011.578282

(C) Vilnius Gediminas Technical University, 2011
www.tandf.co.uk/journals/TMMA

Publisher: Taylor\&Francis and VGTU

Online ISSN: 1648-3510

Print ISSN: 1392-6292

\title{
Reconstruction of a Source Term in a Parabolic Integro-Differential Equation from Final Data*
}

\author{
Kairi Kasemets and Jaan Janno \\ Tallinn University of Technology \\ Ehitajate tee 5, 19086 Tallinn, Estonia \\ E-mail: kairik@staff.ttu.ee \\ E-mail(corresp.): janno@ioc.ee
}

Received November 30, 2010; revised March 31, 2011; published online May 1, 2011

\begin{abstract}
The identification of a source term in a parabolic integro-differential equation is considered. We study the existence of the quasi-solution to this problem, Tikhonov regularization and a related gradient method.
\end{abstract}

Keywords: Inverse problem, integro-differential equation, quasi-solution.

AMS Subject Classification: 35R30; 80A23.

\section{Introduction}

Heat flow processes in media with memory are governed by parabolic integrodifferential equations [7]. A number of papers is devoted to inverse problems to determine kernels of these equations in different formulations making use of measurements over time (see e.g. $[4,6,7,8,11,13,14]$ ).

Recently some papers appeared that deal with the reconstruction of source terms or coefficients of these equations making use of final or integral overdetermination [5, 12]. In particular, the authors' paper [5] extends former existence and uniqueness results of Isakov [3] to the integro-differential case. The existence of the solutions to the inverse problems to determine unknown source terms from final over-determination of the temperature requires sufficient regularity and a certain monotonicity of a time-component of this term.

In the present paper we follow another approach. Instead of the conventional solution, we deal with the quasi-solution of the inverse problem that uses final data. Then we can build up a theory without any smoothness or monotonicity restrictions on the source. Similar results in the case of the parabolic differential equation without an integral term in the one-dimensional case were obtained by Hasanov [2]. Quasi-solutions of other integro-differential inverse problems were studied in $[1,9]$.

\footnotetext{
* Supported by Estonian Science Foundation grant 7728 and Estonian Ministry of Education and Science TF themes SF0140011s09, SF0140083s08.
} 


\section{Direct Problem}

Let $\Omega$ be a $n$-dimensional domain with sufficiently smooth boundary $\Gamma$ and $\Gamma=\Gamma_{1} \cup \Gamma_{2}$ where meas $\Gamma_{1} \cap \Gamma_{2}=0$. Assume that for any $j \in\{1 ; 2\}$ it holds either $\Gamma_{j}=\emptyset$ or meas $\Gamma_{j}>0$. Denote $\Omega_{T}=\Omega \times(0, T), \Gamma_{1, T}=\Gamma_{1} \times(0, T)$, $\Gamma_{2, T}=\Gamma_{2} \times(0, T)$. Consider the problem (direct problem) to find $u(x, t)$ : $\Omega_{T} \rightarrow \mathbb{R}$ such that

$$
\begin{aligned}
& u_{t}=A u-m * A u+f+\nabla \phi \quad \text { in } \Omega_{T}, \\
& u=u_{0} \quad \text { in } \Omega \times\{0\}, \\
& u=g \quad \text { in } \Gamma_{1, T}, \\
& -\nu_{A} \cdot \nabla u+m * \nu_{A} \cdot \nabla u=\vartheta u+h \text { in } \Gamma_{2, T}
\end{aligned}
$$

where

$$
\begin{aligned}
& A v=\sum_{i, j=1}^{n} \frac{\partial}{\partial x_{i}}\left(a_{i j} \frac{\partial}{\partial x_{j}} v\right)+a v, \\
& \nu_{A}=\sum_{j=1}^{n} a_{i j} \nu_{j}, \quad \nu=\left(\nu_{1}, \ldots, \nu_{n}\right) \text { - outer normal of } \Gamma_{2},
\end{aligned}
$$

$a_{i j}, a, u_{0}: \Omega \rightarrow \mathbb{R}, f: \Omega_{T} \rightarrow \mathbb{R}, \phi: \Omega_{T} \rightarrow \mathbb{R}^{n}, g: \Gamma_{1, T} \rightarrow \mathbb{R}, \vartheta: \Gamma_{2} \rightarrow \mathbb{R}$, $h: \Gamma_{2, T} \rightarrow \mathbb{R}, m:(0, T) \rightarrow \mathbb{R}$ are given functions and

$$
m * w(t)=\int_{0}^{t} m(t-\tau) w(\tau) d \tau
$$

denotes the time convolution. In case $\Gamma_{1}=\emptyset\left(\Gamma_{2}=\emptyset\right)$, the boundary condition (2.3) ((2.4)) is dropped.

The problem (2.1)-(2.4) describes the heat flow in a body $\Omega$ with the thermal memory. Concerning the physical background we refer the reader to [7]. The solution $u$ is the temperature of the body and $m$ is the heat flux relaxation (or memory) kernel. The boundary condition (2.4) is of the third kind where the term $-\nu_{A} \cdot \nabla u+m * \nu_{A} \cdot \nabla u$ equals the heat flux in the direction of the co-normal vector.

Let us introduce some additional notation. Let $X$ be a Banach space. We denote by $C([0, T] ; X)$ the space of abstract continuous functions from $[0, T]$ to $X$ endowed with the usual maximum norm $\|v\|_{C([0, T] ; X)}:=\max _{t \in[0, T]}\|v(x)\|$. Moreover, let

$$
L^{2}((0, T) ; X):=\left\{v:(0, T) \rightarrow X:\|v\|_{L^{2}((0, T) ; X)}=\left[\int_{0}^{T}\|v(t)\|^{2} d t\right]^{1 / 2}<\infty\right\} .
$$

In addition, we need spaces of fractional order and anisotropic spaces. To this end, let us first introduce the following notation for difference quotients of $x$ and $(x, t)$-dependent functions with powers:

$$
\begin{aligned}
\langle v\rangle_{p}\left(x_{1}, x_{2}\right):= & \frac{v\left(x_{1}\right)-v\left(x_{2}\right)}{\left|x_{1}-x_{2}\right|^{p}}, \quad\langle v\rangle_{p}\left(x_{1}, x_{2} ; t\right):=\frac{v\left(x_{1}, t\right)-v\left(x_{2}, t\right)}{\left|x_{1}-x_{2}\right|^{p}}, \\
& \langle v\rangle_{p}\left(x ; t_{1}, t_{2}\right):=\frac{v\left(x, t_{1}\right)-v\left(x, t_{2}\right)}{\left|t_{1}-t_{2}\right|^{p}},
\end{aligned}
$$


where $|x|$ denotes the Euclidean norm of $x$ in the space $\mathbb{R}^{n}$. For any $l \geq 0$ we introduce the Sobolev-Slobodeckij spaces (cf. [10, 15])

$$
\begin{aligned}
& W_{2}^{l}(\Omega)=\left\{v:\|v\|_{W_{2}^{l}(\Omega)}:=\sum_{|\alpha| \leq[l]}\left[\int_{\Omega}\left|D_{x}^{\alpha} v(x)\right|^{2} d x\right]^{\frac{1}{2}}\right. \\
&\left.+\Theta_{l} \sum_{|\alpha|=[l]}\left[\int_{\Omega} d x_{1} \int_{\Omega}\left|\left\langle D_{x}^{\alpha} v\right\rangle_{\frac{n}{2}+l-[l]}\left(x_{1}, x_{2}\right)\right|^{2} d x_{2}\right]^{\frac{1}{2}}<\infty\right\}, \\
& W_{2}^{l, \frac{l}{2}}\left(\Omega_{T}\right)=\left\{v:\|v\|_{W_{2}^{l, \frac{l}{2}}\left(\Omega_{T}\right)}:=\sum_{2 j+|\alpha| \leq[l]}\left[\int_{0}^{T} \int_{\Omega}\left|D_{t}^{j} D_{x}^{\alpha} v(x, t)\right|^{2} d x d t\right]^{\frac{1}{2}}\right. \\
&+ \Theta_{l} \sum_{2 j+|\alpha|=[l]}\left[\int_{0}^{T} d t \int_{\Omega} d x_{1} \int_{\Omega}\left|\left\langle D_{t}^{j} D_{x}^{\alpha} v\right\rangle_{\frac{n}{2}+l-[l]}\left(x_{1}, x_{2} ; t\right)\right|^{2} d x_{2}\right]^{\frac{1}{2}} \\
&+\Theta_{\frac{l}{2}} \sum_{\substack{l-2 j-|\alpha| \\
\in(0,2)}}\left[\int_{\Omega} d x \int_{0}^{T} d t_{1} \int_{0}^{T} \mid\left\langle D_{t}^{j} D_{x}^{\alpha} v\right\rangle_{\left.\left.\frac{1}{2}+\left.\frac{l-2 j-|\alpha|}{2}\left(x ; t_{1}, t_{2}\right)\right|^{2} d t_{2}\right]^{\frac{1}{2}}<\infty\right\} .}\right.
\end{aligned}
$$

Here $\alpha=\left(\alpha_{1}, \ldots, \alpha_{n}\right)$ with $\alpha_{i} \in\{0,1,2, \ldots\}$ is the multi-index, $|\alpha|=\alpha_{1}+$ $\cdots+\alpha_{n}, D_{x}^{\alpha} v=\frac{\partial^{|\alpha|} v}{\partial x_{1}^{\alpha_{1}} \cdots \partial x_{n}^{\alpha_{n}}}$ and $D_{t}^{j} v=\frac{\partial^{j} v}{\partial t^{j}}$. Moreover, [l] is the greatest integer $\leq l$ and $\Theta_{l}=0$ and $\Theta_{l}=1$ in the cases of integer $l$ and non-integer $l$, respectively. The definition of $W_{2}^{l, \frac{l}{2}}$ is in a standard manner extended from $\Omega_{T}$ to the boundary components $\Gamma_{1, T}$ and $\Gamma_{2, T}$ (for details see [10]).

Now we return to the direct problem (2.1)-(2.4). Throughout the paper we assume the following basic regularity conditions on the coefficients, the kernel and the initial and boundary functions:

$$
\begin{aligned}
& a_{i j} \in C^{1}(\bar{\Omega}), \quad a_{i j}=a_{j i}, \quad a \in C(\bar{\Omega}), \quad \vartheta \in C\left(\bar{\Gamma}_{2}\right), \quad \vartheta \geq 0, \\
& m \in L^{1}(0, T), \quad g \in W_{2}^{\frac{1}{2}, \frac{1}{4}}\left(\Gamma_{1, T}\right), \quad h \in L^{2}\left(\Gamma_{2, T}\right), \\
& u_{0} \in L^{2}(\Omega), \quad f \in L^{2}\left(\Omega_{T}\right), \quad \phi=\left(\phi_{1}, \ldots, \phi_{n}\right) \in\left(L^{2}\left(\Omega_{T}\right)\right)^{n}
\end{aligned}
$$

and the ellipticity condition

$$
\sum_{i, j=1}^{n} a_{i j} \lambda_{i} \lambda_{i} \geq \epsilon|\lambda|^{2}, \quad x \in \bar{\Omega}, \lambda \in \mathbb{R}^{n} \text { with some } \epsilon>0 .
$$

The first aim is to reformulate the problem (2.1)-(2.4) in a weak form. Let us suppose that (2.1)-(2.4) has a classical solution $u \in W_{2}^{2,1}\left(\Omega_{T}\right)$ and the term $\phi$ satisfies the following additional conditions: $\frac{\partial}{\partial x_{i}} \phi_{i} \in\left(L^{2}\left(\Omega_{T}\right)\right)^{n}, i=1, \ldots, n$, $\left.\phi\right|_{\Gamma_{2, T}}=0$. Then, we multiply (2.1) with a test function $\eta$ from the space

$$
\begin{gathered}
\mathcal{T}\left(\Omega_{T}\right)=\left\{\eta \in L^{2}\left((0, T) ; W_{2}^{1}(\Omega)\right): \eta_{t} \in L^{2}\left((0, T) ; L^{2}(\Omega)\right),\right. \\
\left.\left.\eta\right|_{\Gamma_{1}}=0 \text { in case } \Gamma_{1} \neq \emptyset\right\}
\end{gathered}
$$


and integrate by parts with respect to time and space variables. We obtain the following relation:

$$
\begin{aligned}
0= & \int_{\Omega}\left[u(x, T) \eta(x, T)-u_{0}(x) \eta(x, 0)\right] d x-\iint_{\Omega_{T}} u \eta_{t} d x d t \\
& +\iint_{\Omega_{T}}\left[\sum_{i, j=1}^{n} a_{i j}\left(u_{x_{j}}-m * u_{x_{j}}\right) \eta_{x_{i}}-a(u-m * u) \eta\right] d x d t \\
& +\iint_{\Gamma_{2}, T}(\vartheta u+h) \eta d \Gamma d t-\iint_{\Omega_{T}}(f \eta-\phi \cdot \nabla \eta) d x d t .
\end{aligned}
$$

This relation makes sense also in a more general case when $\phi$ satisfies only (2.7) and $u$ doesn't have regular first order time and second order spatial derivatives. We call a weak solution of the problem (2.1)-(2.4) a function from the space

$$
\mathcal{U}\left(\Omega_{T}\right)=C\left([0, T] ; L^{2}(\Omega)\right) \cap L^{2}\left((0, T) ; W_{2}^{1}(\Omega)\right)
$$

that satisfies the relation $(2.9)$ for any $\eta \in \mathcal{T}\left(\Omega_{T}\right)$ and in case $\Gamma_{1} \neq \emptyset$ fulfills the boundary condition (2.3).

Theorem 1. The problem (2.1)-(2.4) has a unique weak solution. If, in addition, $\phi=0, g \in W_{2}^{\frac{3}{2}, \frac{3}{4}}\left(\Gamma_{1, T}\right), h \in W_{2}^{\frac{1}{2}, \frac{1}{4}}\left(\Gamma_{2, T}\right), u_{0} \in H^{1}(\Omega)$ and $u_{0}=g$ on $\Gamma_{1} \times\{0\}$ then this solution belongs to the space $W_{2}^{2,1}\left(\Omega_{T}\right)$ and satisfies (2.1)-(2.4) in the classical sense.

Proof. It is well known (see e.g. [10]) that in the particular case $m=0$ the solution exists, is unique and the operator $\mathcal{H}$, that assigns to the data vector $u_{0}, g, h, f, \phi$ the weak solution is Lipschitz-continuous from the space $L^{2}(\Omega) \times W_{2}^{\frac{1}{2}, \frac{1}{4}}\left(\Gamma_{1, T}\right) \times L^{2}\left(\Gamma_{2, T}\right) \times L^{2}\left(\Omega_{T}\right)^{n+1}$ to the space $\mathcal{U}\left(\Omega_{T}\right)$. Let us denote $\mathcal{G}(f, \phi)=\mathcal{H}(0,0,0, f, \phi)$. Then, denoting by $\widehat{u}$ the solution corresponding to $m=0$, the problem $(2.1)-(2.4)$ for $u$ is in $\mathcal{U}\left(\Omega_{T}\right)$ equivalent to the following operator equation for the function $v=u-\widehat{u}$ :

$$
v=\mathcal{F} \widehat{u}+\mathcal{F} v
$$

with the linear operator $\mathcal{F} v=\mathcal{G}\left(-m *(a v),-m *\left(\sum_{j=1}^{n} a_{i j} v_{x_{j}}\right)\right)$. We are going to estimate $\mathcal{F}$. To this end, we make use of the following inequality that immediately follows from the estimate (19) in [5]:

$$
\|m * w\|_{L^{2}\left(\Omega_{t}\right)} \leq \int_{0}^{t}|m(t-\tau)|\|w\|_{L^{2}\left(\Omega_{\tau}\right)} d \tau, \quad t \in(0, T) .
$$

Here $\Omega_{t}=\Omega \times(0, t)$ for $t \in(0, T)$ and $w$ is an arbitrary element of $L^{2}\left(\Omega_{T}\right)$. Moreover, we define the cutting operator $P_{t}$ by the formula

$$
P_{t} w= \begin{cases}w & \text { in } \Omega_{t}, \\ 0 & \text { in } \Omega_{T} \backslash \Omega_{t} .\end{cases}
$$


Note that it holds $\mathcal{G}\left(P_{t} f, P_{t} \phi\right)(x, t)=\mathcal{G}(f, \phi)(x, t)$ for any $(x, t) \in \Omega_{t}$. Therefore, observing the Lipschitz-continuity of $\mathcal{G}$ and (2.11) we can estimate as follows:

$$
\begin{aligned}
\|\mathcal{F} v\|_{\mathcal{U}\left(\Omega_{t}\right)} & =\left\|\mathcal{G}\left(-m *(a v),-m *\left(\sum_{j=1}^{n} a_{i j} v_{x_{j}}\right)\right)\right\|_{\mathcal{U}\left(\Omega_{t}\right)} \\
& =\left\|\mathcal{G}\left(-P_{t}[m *(a v)],-P_{t}\left[m *\left(\sum_{j=1}^{n} a_{i j} v_{x_{j}}\right)\right]\right)\right\|_{\mathcal{U}\left(\Omega_{t}\right)} \\
& \leq\left\|\mathcal{G}\left(-P_{t}[m *(a v)],-P_{t}\left[m *\left(\sum_{j=1}^{n} a_{i j} v_{x_{j}}\right)\right]\right)\right\|_{\mathcal{U}\left(\Omega_{T}\right)} \\
& \leq C_{1}\left[\left\|P_{t}[m *(a v)]\right\|_{L^{2}\left(\Omega_{T}\right)}+\sum_{i=1}^{n}\left\|P_{t}\left[m * \sum_{j=1}^{n} a_{i j} v_{x_{j}}\right]\right\|_{L^{2}\left(\Omega_{T}\right)}\right] \\
& =C_{1}\left[\|m *(a v)\|\left\|_{L^{2}\left(\Omega_{t}\right)}+\sum_{i=1}^{n}\right\| m * \sum_{j=1}^{n} a_{i j} v_{x_{j}} \|_{L^{2}\left(\Omega_{t}\right)}\right] \\
& \leq C_{2} \int_{0}^{t}|m(t-\tau)|\left(\|v\|_{L^{2}\left(\Omega_{\tau}\right)}+\|\nabla v\|_{L^{2}\left(\Omega_{\tau}\right)}\right) d \tau \\
& \leq C_{2} \int_{0}^{t}|m(t-\tau)|\|v\|_{\mathcal{U}\left(\Omega_{\tau}\right)} d \tau
\end{aligned}
$$

for any $t \in(0, T)$ with some constants $C_{1}, C_{2}$. Now we introduce the weighted norms in $\mathcal{U}\left(\Omega_{T}\right):\|v\|_{\sigma}=\sup _{0<t<T} e^{-\sigma t}\|v\|_{\mathcal{U}\left(\Omega_{t}\right)}$ where $\sigma \geq 0$. Using the deduced estimate for $\mathcal{F}$ we obtain

$$
\begin{aligned}
\|\mathcal{F} v\|_{\sigma} & \leq C_{2} \sup _{0<t<T} e^{-\sigma t} \int_{0}^{t}|m(t-\tau)|\|v\|_{\mathcal{U}\left(\Omega_{\tau}\right)} d \tau \\
& =C_{2} \sup _{0<t<T} \int_{0}^{t} e^{-\sigma(t-\tau)}|m(t-\tau)| e^{-\sigma \tau}\|v\|_{\mathcal{U}\left(\Omega_{\tau}\right)} d \tau \\
& \leq C_{2} \int_{0}^{T} e^{-\sigma s}|m(s)| d s\|v\|_{\sigma} .
\end{aligned}
$$

Since $\int_{0}^{T} e^{-\sigma s}|m(s)| d s \rightarrow 0$ as $\sigma \rightarrow \infty$, the operator $\mathcal{F}$ is a contraction for sufficiently large $\sigma$. Consequently, $(2.10)$ has a unique solution in $\mathcal{U}\left(\Omega_{T}\right)$. This proves the existence of the unique weak solution of $(2.1)-(2.4)$.

Secondly, let us prove the classical solvability assertion of the theorem. Again, we use the results in case $m=0$. It is known [15] that in case $m=0$ the solution belongs to $W_{2}^{1}\left(\Omega_{T}\right)$ and the operator $\mathcal{H}^{1}$ that assigns to the data vector $u_{0}, g, h, f$ the classical solution is Lipschitz-continuous from the space $H^{1}(\Omega) \times W_{2}^{\frac{3}{2}, \frac{3}{4}}\left(\Gamma_{1, T}\right) \times W_{2}^{\frac{1}{2}, \frac{1}{4}}\left(\Gamma_{2, T}\right) \times L^{2}\left(\Omega_{T}\right)$ to the space $W_{2}^{2,1}\left(\Omega_{T}\right)$. Define $\mathcal{G}^{1}(h, f)=\mathcal{H}^{1}(0,0, h, f)$. The problem for $u$ is equivalent to the following operator equation for $v=u-\widehat{u}$ :

$$
v=\mathcal{F}^{1} \widehat{u}+\mathcal{F}^{1} v,
$$


where $\mathcal{F}^{1} v=\mathcal{G}^{1}\left(-\left.m * \nu_{A} \cdot \nabla v\right|_{\Gamma_{2, T}},-m * A v\right)$. This time we have to introduce a more complicated extension operator instead of $P_{t}$ because the argument of $\mathcal{F}^{1}$ has traces on slices $\Omega \times\{t\}$. Let us define

$$
\widetilde{P}_{t} w(x, s)= \begin{cases}w(x, s) & \text { for } s<t \\ w(x, 2 t-s) & \text { for } t<s<\min \{2 t ; T\} \\ 0 & \text { for } s>2 t \text { in case } 2 t<T\end{cases}
$$

Then, since the function $v$ in the range of $\mathcal{F}^{1}$ satisfies $\left.v\right|_{t=0}=0$, it holds $\widetilde{P}_{t} v \in$ $W_{2}^{2,1}\left(\Omega_{T}\right)$ for $t \in(0, T)$. Moreover, $\mathcal{G}^{1}\left(\widetilde{P}_{t} \widetilde{h}, \widetilde{P}_{t} \widetilde{f}\right)(x, t)=\mathcal{G}^{1}(\widetilde{h}, \widetilde{f})(x, t)$ for any $(x, t) \in \Omega_{t}$ and $\left\|\widetilde{P_{t}} \widetilde{h}\right\|_{W_{2}^{\frac{1}{2}, \frac{1}{4}}\left(\Gamma_{2, T}\right)} \leq 2\|\widetilde{h}\|_{W_{2}^{\frac{1}{2}}, \frac{1}{4}\left(\Gamma_{2, t}\right)},\left\|\widetilde{P}_{t} \widetilde{f}\right\|_{L^{2}\left(\Omega_{T}\right)} \leq 2\|\widetilde{f}\|_{L^{2}\left(\Omega_{t}\right)}$, where $\widetilde{h}=\left.m * \nu_{A} \cdot \nabla v\right|_{\Gamma_{2}, T}$ and $\widetilde{f}=m * A v$. Consequently, in view of the Lipschitz-continuity of $\mathcal{G}^{1}$ we deduce

$$
\begin{aligned}
\left\|\mathcal{F}^{1} v\right\|_{W_{2}^{2,1}\left(\Omega_{t}\right)} & =\left\|\mathcal{G}^{1}\left(-\left.m * \nu_{A} \cdot \nabla v\right|_{\Gamma_{2, T}},-m * A v\right)\right\|_{W_{2}^{2,1}\left(\Omega_{t}\right)} \\
& =\left\|\mathcal{G}^{1}\left(-P_{t}\left[\left.m * \nu_{A} \cdot \nabla v\right|_{\Gamma_{2, T}}\right],-P_{t}[m * A v]\right)\right\|_{W_{2}^{2,1}\left(\Omega_{t}\right)} \\
& \leq\left\|\mathcal{G}^{1}\left(-P_{t}\left[\left.m * \nu_{A} \cdot \nabla v\right|_{\Gamma_{2, T}}\right],-P_{t}[m * A v]\right)\right\|_{W_{2}^{2,1}\left(\Omega_{T}\right)} \\
& \leq C_{3}\left[\left\|P_{t}\left[m * \nu_{A} \cdot \nabla v\right]\right\|_{W_{2}^{\frac{1}{2}, \frac{1}{4}}\left(\Gamma_{2, T}\right)}+\left\|P_{t}[m * A v]\right\|_{L^{2}\left(\Omega_{T}\right)}\right] \\
& \leq 2 C_{3}\left[\left\|m * \nu_{A} \cdot \nabla v\right\|_{W_{2}^{\frac{1}{2}, \frac{1}{4}}\left(\Gamma_{2, t}\right)}+\|m * A v\|_{L^{2}\left(\Omega_{t}\right)}\right]
\end{aligned}
$$

for any $t \in(0, T)$ with some constant $C_{3}$ and $\Gamma_{2, t}=\Gamma_{2} \times(0, t)$. Using the trace theorem for Sobolev-Slobodeckij spaces [10] and the relation $(m * v)_{t}=m * v_{t}$, that holds due to $\left.v\right|_{t=0}=0$, we compute

$$
\begin{aligned}
\left\|m * \nu_{A} \cdot \nabla v\right\|_{W_{2}^{\frac{1}{2}, \frac{1}{4}}\left(\Gamma_{2, t}\right)} & =\left\|\nu_{A} \cdot \nabla(m * v)\right\|_{W_{2}^{\frac{1}{2}, \frac{1}{4}}\left(\Gamma_{2, t}\right)} \leq C_{4}\|m * v\|_{W_{2}^{2,1}\left(\Omega_{t}\right)} \\
& =C_{4}\left[\sum_{|\alpha| \leq 2}\left\|m * D_{x}^{\alpha} v\right\|_{L^{2}\left(\Omega_{t}\right)}+\left\|m * v_{t}\right\|_{L^{2}\left(\Omega_{t}\right)}\right]
\end{aligned}
$$

with some constant $C_{4}$. Applying this estimate in (2.13) and using (2.11) we deduce

$$
\left\|\mathcal{F}^{1} v\right\|_{W_{2}^{2,1}\left(\Omega_{t}\right)} \leq C_{5} \int_{0}^{t}|m(t-\tau)|\|v\|_{W_{2}^{2,1}\left(\Omega_{\tau}\right)} d \tau, \quad t \in(0, T)
$$

with a constant $C_{5}$. We define the weighted norms

$$
\|v\|_{\sigma}^{*}=\sup _{0<t<T} e^{-\sigma t}\|v\|_{W_{2}^{2,1}\left(\Omega_{t}\right)}
$$

in the space $W_{2}^{2,1}\left(\Omega_{T}\right)$ and, as in the first part of the proof, show that $\mathcal{F}^{1}$ is a contraction in $W_{2}^{2,1}\left(\Omega_{T}\right)$ if $\sigma$ is sufficiently large. This proves the unique solvability of (2.12) and in turn the classical solvability assertion of theorem. 


\section{Formulation of Inverse Problem. Existence of Quasi- Solution}

Let $\widehat{\mathcal{F}}$ be a linear closed subspace of $L^{2}\left(\Omega_{T}\right)$. Suppose that the source term $f$ is of the following form: $f=f_{0}+F$, where $f_{0} \in L^{2}\left(\Omega_{T}\right)$ is known. We pose an inverse problem to determine the function $F \in \widehat{\mathcal{F}}$ making use of the final measurement

$$
u(x, T)=u_{T}(x), \quad x \in \Omega .
$$

More precisely, we will search a quasi-solution of this problem. This is a solution of the following minimization problem for the cost functional: find

$$
F^{*}=\arg \min _{F \in \mathcal{F}} J(F), \quad J(F)=\left\|u(\cdot, T ; F)-u_{T}\right\|_{L^{2}(\Omega)}^{2},
$$

where $\mathcal{F} \subseteq \widehat{\mathcal{F}}$ is a subset including constraints. Here $u(x, t ; F)$ stands for the solution of the direct problem corresponding to the given $F$.

Let us introduce some cases of $\widehat{\mathcal{F}}$.

Case 1. Define $\widehat{\mathcal{F}}=\left\{F: F(x, t)=\varkappa(t) w(x), w \in L^{2}(\Omega)\right\}$, where $\varkappa \in L^{2}(0, T)$, $\varkappa \neq 0$ is a prescribed function.

Case 2. Let $\Omega$ be a cylinder: $\Omega=S \times(0, l)$, where for any $x=\left(x_{1}, \ldots, x_{n}\right) \in \Omega$ we have $\bar{x}=\left(x_{1}, \ldots, x_{n-1}\right) \in S, x_{n} \in(0, l)$. Define $\widehat{\mathcal{F}}=\{F: F(x, t)=$ $\left.\varkappa\left(x_{n}\right) w(\bar{x}, t), w \in L^{2}\left(S_{T}\right)\right\}$, where $\varkappa \in L^{2}(0, l), \varkappa \neq 0$ is a prescribed function and $S_{T}=S \times(0, T)$.

Case 3. Define $\widehat{\mathcal{F}}=\left\{F: F(x, t)=\sum_{j=1}^{N} w_{j} \varkappa_{j}(x, t), w=\left(w_{j}\right)_{j=1, \ldots, N} \in \mathbb{R}^{N}\right\}$, where $\varkappa=\left(\varkappa_{j}\right)_{j=1, \ldots, N} \in\left(L^{2}\left(\Omega_{T}\right)\right)^{N}, \varkappa \neq 0$ is a prescribed vectorfunction. In practice, the component $\varkappa_{j}$ may be the characteristic function of a subdomain $\Omega_{j} \subset \Omega$.

Now let us consider the first variation of the cost functional

$$
\begin{aligned}
& \Delta J(F)=J(F+\Delta F)-J(F) \\
& \quad=2 \int_{\Omega}\left[u(x, T ; F)-u_{T}(x)\right] \Delta u(x, T ; F) d x+\int_{\Omega}[\Delta u(x, T ; F)]^{2} d x,
\end{aligned}
$$

where $\Delta u(x, t ; F)=u(x, t ; F+\Delta F)-u(x, t ; F)$. By Theorem 1 , the function $\Delta u$ belongs to $W_{2}^{2,1}\left(\Omega_{T}\right)$ and solves the following problem in the classical sense:

$$
\begin{aligned}
& \Delta u_{t}=A \Delta u-m * A \Delta u+\Delta F \text { in } \Omega_{T}, \\
& \Delta u=0 \quad \text { in } \Omega \times\{0\}, \\
& \Delta u=0 \quad \text { in } \Gamma_{1, T}, \\
& -\nu_{A} \cdot \nabla \Delta u+m * \nu_{A} \cdot \nabla \Delta u=\vartheta \Delta u \text { in } \Gamma_{2, T} .
\end{aligned}
$$

Moreover, let us introduce the following adjoint problem with the solution $\psi(x, t ; F)$ :

$$
\begin{aligned}
& \psi_{t}(x, t ; F)=-A \psi(x, t ; F)+\int_{t}^{T} m(\tau-t) A \psi(x, \tau ; F) d \tau \text { in } \Omega_{T}, \\
& \psi(x, T ; F)=2\left[u(x, T ; F)-u_{T}(x)\right] \quad \text { in } \Omega,
\end{aligned}
$$




$$
\begin{aligned}
& \psi(x, t ; F)=0 \quad \text { in } \Gamma_{1, T} \\
& -\nu_{A} \cdot \nabla \psi(x, t ; F)+\int_{t}^{T} m(\tau-t) \nu_{A} \cdot \nabla \psi(x, \tau ; F) d \tau=\vartheta \psi(x, t ; F) \quad \text { in } \Gamma_{2, T}
\end{aligned}
$$

It is easy to see that the equivalent problem for $\tilde{u}(x, t)=\psi(x, T-t ; F)$ is of the form (2.1)-(2.4) with homogeneous differential equation and boundary conditions and the initial condition $\tilde{u}=2\left[u(\cdot, T ; F)-u_{T}\right] \in L^{2}(\Omega)$ in $\Omega \times\{0\}$. Therefore, applying Theorem 1 we conclude that problem (3.7)-(3.10) has a unique weak solution. The weak problem for $\psi(x, T-t ; F)$ reads

$$
\begin{aligned}
0= & \int_{\Omega}\left[\psi(x, 0 ; F) \eta(x, T)-2\left[u(x, T ; F)-u_{T}(x)\right] \eta(x, 0)\right] d x \\
& -\iint_{\Omega_{T}} \psi(x, T-t ; F) \eta_{t}(x, t) d x d t+\iint_{\Omega_{T}}\left[\sum _ { i , j = 1 } ^ { n } a _ { i j } ( x ) \left(\psi_{x_{j}}(x, T-t ; F)\right.\right. \\
& \left.-\int_{0}^{t} m(t-\tau) \psi_{x_{j}}(x, T-\tau ; F) d \tau\right) \eta_{x_{i}}(x, t) \\
& \left.-a(x)\left(\psi(x, T-t ; F)-\int_{0}^{t} m(t-\tau) \psi(x, T-\tau ; F) d \tau\right) \eta(x, t)\right] d x d t \\
& +\int_{\Gamma_{2}, T} \vartheta \psi(x, T-t ; F) \eta(x, t) d \Gamma d t \quad \forall \eta \in \mathcal{T}\left(\Omega_{T}\right) .
\end{aligned}
$$

Lemma 1. It holds the following formula:

$$
2 \int_{\Omega}\left[u(x, T ; F)-u_{T}(x)\right] \Delta u(x, T, F) d x=\iint_{\Omega_{T}} \psi(x, t ; F) \Delta F(x, t) d x d t .
$$

Proof. Since $\Delta u \in W_{2}^{2,1}\left(\Omega_{T}\right)$ satisfies the homogeneous boundary condition on $\Gamma_{1}$, it holds $\Delta u(x, T-t, F) \in \mathcal{T}\left(\Omega_{T}\right)$. Let us use the test function $\eta(x, t)=$ $\Delta u(x, T-t, F)$ in (3.11). This yields (changing the variable $t$ by $T-t$ under the integrals and observing that $\eta(x, T)=0$ and omitting $F$ in the arguments for the sake of shortness)

$$
\begin{aligned}
0= & \left.-2 \int_{\Omega}\left[u(x, T)-u_{T}(x)\right] \Delta u(x, T)\right] d x+\iint_{\Omega_{T}} \psi(x, t) \Delta u_{t}(x, t) d x d t \\
& +\iint_{\Omega_{T}}\left[\sum_{i, j=1}^{n} a_{i j}\left(\psi_{x_{j}}(x, t)-\int_{0}^{t} m(t-\tau) \psi_{x_{j}}(x, \tau) d \tau\right) \Delta u_{x_{j}}(x, t)\right. \\
& \left.-a(x)\left(\psi(x, t)-\int_{0}^{t} m(t-\tau) \psi(x, \tau) d \tau\right) \Delta u(x, t)\right] d x d t \\
& +\int_{\Gamma_{2}, T} \vartheta \psi(x, t) \Delta u(x, t) d \Gamma d t .
\end{aligned}
$$


On the other hand, the problem (3.3)-(3.6) in the weak form reads

$$
\begin{aligned}
0= & \int_{\Omega} \Delta u(x, T) \zeta(x, T) d x-\iint_{\Omega_{T}} \Delta u \zeta_{t} d x d t \\
& +\iint_{\Omega_{T}}\left[\sum_{i, j=1}^{n} a_{i j}\left(\Delta u_{x_{j}}-m * \Delta u_{x_{j}}\right) \zeta_{x_{i}}-a(\Delta u-m * \Delta u) \zeta\right] d x d t \\
& +\iint_{\Gamma_{2}, T} \vartheta \Delta u \zeta d \Gamma d t-\iint_{\Omega_{T}} \Delta F \zeta d x d t \quad \forall \zeta \in \mathcal{T}\left(\Omega_{T}\right)
\end{aligned}
$$

Since $\Delta u \in W_{2}^{2,1}\left(\Omega_{T}\right)$ has the regular time derivative, we can integrate by parts the integral $\iint_{\Omega_{T}} \Delta u \zeta_{t} d x d t$ in (3.14). This results in the relation

$$
\begin{aligned}
0= & \iint_{\Omega_{T}} \Delta u_{t} \zeta d x d t+\iint_{\Omega_{T}}\left[\sum_{i, j=1}^{n} a_{i j}\left(\Delta u_{x_{j}}-m * \Delta u_{x_{j}}\right) \zeta_{x_{i}}\right. \\
& -a(\Delta u-m * \Delta u) \zeta] d x d t+\iint_{\Gamma_{2, T}} \vartheta \Delta u \zeta d \Gamma d t-\iint_{\Omega_{T}} \Delta F \zeta d x d t .
\end{aligned}
$$

It is important that this relation doesn't contain the time derivative of the test function $\zeta$. Therefore, we can extend the set of test functions of (3.15) from $\mathcal{T}\left(\Omega_{T}\right)$ to $\mathcal{U}_{0}\left(\Omega_{T}\right)=\left\{\zeta \in \mathcal{U}\left(\Omega_{T}\right):\left.\zeta\right|_{\Gamma_{1, T}}=0\right.$ in case $\left.\Gamma_{2} \neq \emptyset\right\}$. In particular, it is possible to take the test function $\zeta=\psi \in \mathcal{U}_{0}\left(\Omega_{T}\right)$. Then we obtain

$$
\begin{aligned}
0= & \iint_{\Omega_{T}} \Delta u_{t} \psi d x d t+\iint_{\Omega_{T}}\left[\sum_{i, j=1}^{n} a_{i j}\left(\Delta u_{x_{j}}-m * \Delta u_{x_{j}}\right) \psi_{x_{i}}\right. \\
& -a(\Delta u-m * \Delta u) \psi] d x d t+\iint_{\Gamma_{2, T}} \vartheta \Delta u \psi d \Gamma d t-\iint_{\Omega_{T}} \Delta F \psi d x d t .
\end{aligned}
$$

Subtracting (3.16) from (3.13) and changing the order of integration in convolution terms we deduce the formula (3.12). Lemma is proved.

Theorem 2. Let $\mathcal{F}$ be a bounded, closed and convex subset of $\widehat{\mathcal{F}}$. Then the problem (3.1) has a solution in $\mathcal{F}$. Moreover, the set of all solutions $\mathcal{F}^{*}$ form a closed convex subset of $\mathcal{F}$.

Proof. The assertion follows from Weierstrass existence theorem (see [16, Section 2.5]) once we have proved that $J(F)$ is weakly sequentially lower semicontinuous in $\mathcal{F}$, i.e.

$$
J(F) \leq \liminf _{n \rightarrow \infty} J\left(F_{n}\right) \quad \text { as } F_{n} \rightarrow F \text { in } \mathcal{F}
$$

and convex, i.e.

$$
J\left(\gamma F_{1}+(1-\gamma) F_{2}\right) \leq \gamma J\left(F_{1}\right)+(1-\gamma) J\left(F_{2}\right) \quad \forall \gamma \in[0,1], F_{1}, F_{2} \in \mathcal{F}
$$


Let us compute:

$$
\begin{aligned}
J(F)= & \int_{\Omega}\left[u(x, T ; F)-u_{T}(x)\right]^{2} d x=\int_{\Omega}\left[u\left(x, T ; F_{n}\right)-u_{T}(x)\right]^{2} d x \\
& -\int_{\Omega}\left[u\left(x, T ; F_{n}\right)-u(x, T ; F)\right]^{2} d x \\
& -2 \int_{\Omega}\left[u(x, T ; F)-u_{T}(x)\right]\left[u\left(x, T ; F_{n}\right)-u(x, T ; F)\right] d x \\
= & J\left(F_{n}\right)-\int_{\Omega}\left[u\left(x, T ; F_{n}\right)-u(x, T ; F)\right]^{2} d x \\
& -2 \int_{\Omega}\left[u(x, T ; F)-u_{T}(x)\right] \Delta u_{n}(x, T ; F) d x
\end{aligned}
$$

where $\Delta u_{n}(x, t ; F)=u\left(x, T ; F_{n}\right)-u(x, T ; F)$ is the change of $u$ corresponding to the change of the free term $\Delta F_{n}=F_{n}-F$. Thus, in view of (3.12) we have

$$
J(F) \leq J\left(F_{n}\right)-\iint_{\Omega_{T}} \psi(x, t ; F) \Delta F_{n}(x, t) d x d t .
$$

Since $\psi \in L^{2}\left(\Omega_{T}\right)$, this implies the relation (3.17). To prove the convexity, we firstly note that

$$
u\left(x, t ; \gamma F_{1}+(1-\gamma) F_{2}\right)=\gamma u\left(x, t ; F_{1}\right)+(1-\gamma) u\left(x, t ; F_{2}\right), \text { for } \gamma \in[0,1] .
$$

Therefore, in view of the convexity of the quadratic function we obtain

$$
\begin{aligned}
J & \left(\gamma F_{1}+(1-\gamma) F_{2}\right)=\int_{0}^{T}\left[u\left(x, T, \gamma F_{1}+(1-\gamma) F_{2}\right)-u_{T}(x)\right]^{2} d x \\
& =\int_{0}^{T}\left[\gamma\left\{u\left(x, T ; F_{1}\right)-u_{T}(x)\right\}+(1-\gamma)\left\{u\left(x, T ; F_{2}\right)-u_{T}(x)\right\}\right]^{2} d x \\
& \leq \gamma \int_{0}^{T}\left[u\left(x, T, F_{1}\right)-u_{T}(x)\right]^{2} d x+(1-\gamma) \int_{0}^{T}\left[u\left(x, T, F_{2}\right)-u_{T}(x)\right]^{2} d x \\
& =\gamma J\left(F_{1}\right)+(1-\gamma) J\left(F_{2}\right) \quad \text { for } \gamma \in[0,1] .
\end{aligned}
$$

This shows the convexity of $J$. Theorem is proved.

Remark 1. In order to prove the existence in an unbounded set $\mathcal{F}$ incl. $\widehat{\mathcal{F}}$, it is sufficient to have the weak coercivity of $J(F)$. This is a difficult problem, because monotonicity methods in general fail for problems in integro-differential PDE. However, the boundedness assumption of $\mathcal{F}$ seems not very restrictive, because in practice some bound for $F$ may be available.

\section{Regularized Problem}

In [5] we proved that in a particular case the solution of the inverse problem under consideration continuously depends on certain derivatives of the data. 
This shows the ill-posedness of the problem in case the data have noise in $L^{2}$ space. We can easily incorporate Tikhonov regularization in quasi-solution. In this case we minimize the stabilized cost functional: find

$$
F^{*}=\arg \min _{F \in \mathcal{F}} J_{\alpha}(F), \quad J_{\alpha}(F)=\alpha\|F\|_{L^{2}\left(\Omega_{T}\right)}^{2}+\left\|u(\cdot, T ; F)-u_{T}\right\|_{L^{2}(\Omega)}^{2} .
$$

Here $\alpha>0$ is the regularization parameter that depends on the noise level of the data $u_{T}$. If we set here $\alpha=0$, we get the original problem (3.1).

Theorem 3. Let $\alpha>0$ and $\mathcal{F}$ be a closed and convex subset of $\widehat{\mathcal{F}}$ (may be also $\mathcal{F}=\widehat{\mathcal{F}})$. Then the problem (4.1) has a unique solution in $\mathcal{F}$.

Proof. Obviously the additional term $I(F)=\alpha\|F\|_{L^{2}\left(\Omega_{T}\right)}$ is strictly convex:

$$
I\left(\gamma F_{1}+(1-\gamma) F_{2}\right)<\gamma I\left(F_{1}\right)+(1-\gamma) I\left(F_{2}\right) \quad \forall \gamma \in(0,1), F_{1}, F_{2} \in \mathcal{F}
$$

and weakly coercive, i.e., $I(F) \rightarrow \infty$ as $\|F\|_{L^{2}\left(\Omega_{T}\right)} \rightarrow \infty$. This makes the whole functional $J_{\alpha}$ strictly convex and weakly coercive. Moreover, it is easy to check that $I(F)$ is weakly sequentially lower semi-continuous. Since $J(F)=$ $\left\|u(\cdot, T ; F)-u_{T}\right\|_{L^{2}(\Omega)}^{2}$ is also weakly lower semi-continuous (this was shown in the proof of Theorem 2), the whole functional $J_{\alpha}$ is weakly lower semicontinuous. Now the assertion of the theorem follows from Weierstrass existence theorem [16, Section 2.5].

\section{$5 \quad$ Auxiliary Estimates}

Lemma 2. The following estimate is valid with a constant $C_{0}$ :

$$
\|\Delta u(\cdot, T ; F)\|_{L^{2}(\Omega)} \leq C_{0}\|\Delta F\|_{L^{2}\left(\Omega_{T}\right)} .
$$

Proof. For the sake of shortness, we omit $F$ in the list of arguments of $\Delta u$. Firstly, we prove this assertion in case $\|m\|_{L^{1}(0, T)}$ is small enough and the equation for $\Delta u(3.3)$ contains an additional term, namely it has the form

$$
\Delta u_{t}=A \Delta u-\sigma \Delta u-m * A \Delta u+\Delta F \quad \text { in } \Omega_{T},
$$

where $\sigma$ is a sufficiently large number such that $\sigma-a(x) \geq \epsilon$ for any $x \in \Omega$. By Theorem 1, $\Delta u$ belongs to $W_{2}^{2,1}\left(\Omega_{T}\right)$ and solves the problem (5.2), (3.4)-(3.6) in the classical sense. Let us multiply the equation (5.2) by $\Delta u$ and integrate by parts taking into account the definition of $A$ and the homogeneous boundary conditions (3.5), (3.6):

$$
\begin{aligned}
0 & =\iint_{\Omega_{T}}\left[\Delta u_{t}-(A-\sigma) \Delta u+m * A \Delta u-\Delta F\right] \Delta u d x d t \\
& =\frac{1}{2} \iint_{\Omega_{T}}\left[\Delta u^{2}\right]_{t} d x d t+\iint_{\Omega_{T}}\left[\sum_{i, j=1}^{n} a_{i j} \Delta u_{x_{j}} \Delta u_{x_{i}}+(\sigma-a) \Delta u^{2}\right] d x d t
\end{aligned}
$$




$$
\begin{aligned}
& -\iint_{\Omega_{T}}\left[\sum_{i, j=1}^{n} a_{i j}\left(m * \Delta u_{x_{j}}\right) \Delta u_{x_{i}}-a(m * \Delta u) \Delta u\right] d x d t \\
& +\iint_{\Gamma_{2}, T} \vartheta \Delta u^{2} d \Gamma d t-\iint_{\Omega_{T}} \Delta F \Delta u d x d t .
\end{aligned}
$$

In view of the homogeneous initial condition (3.4), this relation can be transformed to the form

$$
\begin{aligned}
& \frac{1}{2} \int_{\Omega}[\Delta u(x, T)]^{2} d x+\iint_{\Gamma_{2}, T} \vartheta \Delta u^{2} d \Gamma d t \\
& \quad+\iint_{\Omega_{T}}\left[\sum_{i, j=1}^{n} a_{i j} \Delta u_{x_{j}} \Delta u_{x_{i}}+(\sigma-a) \Delta u^{2}\right] d x d t \\
& \quad=\iint_{\Omega_{T}}\left[\sum_{i, j=1}^{n} a_{i j}\left(m * \Delta u_{x_{j}}\right) \Delta u_{x_{i}}-a(m * \Delta u) \Delta u\right] d x d t+\iint_{\Omega_{T}} \Delta F \Delta u d x d t .
\end{aligned}
$$

Due to the assumptions $\vartheta \geq 0,(2.8)$ and $\sigma-a \geq \epsilon$, the left hand side of (5.3) can be estimated from below:

$$
\begin{aligned}
& \frac{1}{2} \int_{\Omega}[\Delta u(x, T)]^{2} d x+\iint_{\Gamma_{2, T}} \vartheta \Delta u^{2} d x d t \\
& \quad+\iint_{\Omega_{T}}\left[\sum_{i, j=1}^{n} a_{i j} \Delta u_{x_{j}} \Delta u_{x_{i}}+(\sigma-a) \Delta u^{2}\right] d x d t \\
& \geq \frac{1}{2} \int_{\Omega}[\Delta u(x, T)]^{2} d x+\epsilon \iint_{\Omega_{T}}\left[|\nabla \Delta u|^{2}+\Delta u^{2}\right] d x d t=: I^{2} .
\end{aligned}
$$

The right-hand side of (5.3) is estimated from above by means of the CauchySchwarz inequality:

$$
\begin{aligned}
& \iint_{\Omega_{T}} {\left[\sum_{i, j=1}^{n} a_{i j}\left(m * \Delta u_{x_{j}}\right) \Delta u_{x_{i}}-a(m * \Delta u) \Delta u\right] d x d t+\iint_{\Omega_{T}} \Delta F \Delta u d x d t } \\
& \leq \bar{C}_{1}\left[\sum_{i, j=1}^{n}\left\|m * \Delta u_{x_{j}}\right\|_{L^{2}\left(\Omega_{T}\right)}\left\|\Delta u_{x_{i}}\right\|_{L^{2}\left(\Omega_{T}\right)}\right. \\
&\left.\quad+\|m * \Delta u\|_{L^{2}\left(\Omega_{T}\right)}\|\Delta u\|_{L^{2}\left(\Omega_{T}\right)}\right]+\|\Delta F\|_{L^{2}\left(\Omega_{T}\right)}\|\Delta u\|_{L^{2}\left(\Omega_{T}\right)}
\end{aligned}
$$

where $\bar{C}_{1}$ is a constant depending on the coefficients $a_{i j}$ and $a$. For the convolution terms we apply the Young's inequality in the space $L^{2}\left(\Omega_{T}\right)=$ $L^{2}\left((0, T) ; L^{2}(\Omega)\right)$. This yields

$$
\begin{aligned}
& \left\|m * \Delta u_{x_{j}}\right\|_{L^{2}\left(\Omega_{T}\right)} \leq\|m\|_{L^{1}(0, T)}\left\|\Delta u_{x_{j}}\right\|_{L^{2}\left(\Omega_{T}\right)}, \quad j=1, \ldots, n, \\
& \|m * \Delta u\|_{L^{2}\left(\Omega_{T}\right)} \leq\|m\|_{L^{1}(0, T)}\|\Delta u\|_{L^{2}\left(\Omega_{T}\right)} .
\end{aligned}
$$


Using (5.4)-(5.6) in (5.3) we obtain

$$
\begin{aligned}
I^{2} \leq & \bar{C}_{1}\|m\|_{L^{1}(0, T)}\left[\sum_{i, j=1}^{n}\left\|\Delta u_{x_{j}}\right\|_{L^{2}\left(\Omega_{T}\right)}\left\|\Delta u_{x_{i}}\right\|_{L^{2}\left(\Omega_{T}\right)}+\|\Delta u\|_{L^{2}\left(\Omega_{T}\right)}^{2}\right] \\
& +\|\Delta F\|_{L^{2}\left(\Omega_{T}\right)}\|\Delta u\|_{L^{2}\left(\Omega_{T}\right)} .
\end{aligned}
$$

Further, we use the inequalities

$$
\left\|\Delta u_{x_{i}}\right\|_{L^{2}\left(\Omega_{T}\right)} \leq\||\nabla \Delta u|\|_{L^{2}\left(\Omega_{T}\right)}, \quad i=1, \ldots, n,
$$

and definition of $I$ (see (5.4)). We have

$$
\begin{aligned}
I^{2} \leq & \bar{C}_{1}\|m\|_{L^{1}(0, T)}\left[n^{2}\||\nabla \Delta u|\|_{L^{2}\left(\Omega_{T}\right)}^{2}+\|\Delta u\|_{L^{2}\left(\Omega_{T}\right)}^{2}\right] \\
& +\|\Delta F\|_{L^{2}\left(\Omega_{T}\right)}\|\Delta u\|_{L^{2}\left(\Omega_{T}\right)} \leq \frac{\bar{C}_{1} n^{2}\|m\|_{L^{1}(0, T)}}{\epsilon} I^{2}+\frac{1}{\sqrt{\epsilon}}\|\Delta F\|_{L^{2}\left(\Omega_{T}\right)} I .
\end{aligned}
$$

Therefore, in case $m$ satisfies the smallness condition

$$
\|m\|_{L^{1}(0, T)} \leq \frac{\epsilon}{2 \bar{C}_{1} n^{2}},
$$

we obtain $I^{2} \leq \frac{2}{\sqrt{\epsilon}}\|\Delta F\|_{L^{2}\left(\Omega_{T}\right)} I$ that yields $I \leq \frac{2}{\sqrt{\epsilon}}\|\Delta F\|_{L^{2}\left(\Omega_{T}\right)}$. Observing that $\|\Delta u(\cdot, T)\|_{L^{2}(\Omega)} \leq \sqrt{2} I$, from the latter inequality we deduce the estimate (5.1) with the constant $C_{0}=2 \sqrt{2} / \epsilon$.

Now let us return to the original problem (3.3)-(3.6) without the additional $\sigma$-term and arbitrarily large $m$. Define the following function: $\Delta u_{\sigma}(x, t)=$ $e^{-\sigma t} \Delta u(x, t)$ where $\sigma \in \mathbb{R}$. It is easy to check that $\Delta u_{\sigma}$ solves the following problem:

$$
\begin{aligned}
& \Delta u_{\sigma, t}=A \Delta u_{\sigma}-\sigma \Delta u_{\sigma}-m_{\sigma} * A \Delta u_{\sigma}+\Delta F_{\sigma} \quad \text { in } \Omega_{T}, \\
& \Delta u_{\sigma}=0 \quad \text { in } \Omega \times\{0\} \\
& \Delta u_{\sigma}=0 \quad \text { in } \Gamma_{1, T}, \\
& -\nu_{A} \cdot \nabla \Delta u_{\sigma}+m_{\sigma} * \nu_{A} \cdot \nabla \Delta u_{\sigma}=\vartheta \Delta u_{\sigma} \quad \text { in } \Gamma_{2, T}
\end{aligned}
$$

where $m_{\sigma}(t)=e^{-\sigma t} m(t)$ and $\Delta F_{\sigma}(x, t)=e^{-\sigma t} \Delta F(x, t)$. Clearly, there exists a sufficiently large $\sigma$ such that $m_{\sigma}$ satisfies the condition (5.7) and the inequality $\sigma-a(x) \geq \epsilon$ is valid for $x \in \Omega$. Therefore, the first part of the proof applies to the function $\Delta u_{\sigma}$. This means that the estimate

$$
\left\|\Delta u_{\sigma}(\cdot, T)\right\|_{L^{2}(\Omega)} \leq \frac{2 \sqrt{2}}{\epsilon}\left\|\Delta F_{\sigma}\right\|_{L^{2}\left(\Omega_{T}\right)}
$$

is valid. Finally, in view of $\Delta u_{\sigma}(x, T)=e^{-\sigma T} \Delta u(x, T)$ and $\left|\Delta F_{\sigma}(x, t)\right| \leq$ $|\Delta F(x, t)|$, from (5.8) we obtain the desired estimate (5.1) with the constant $C_{0}=2 \sqrt{2} e^{\sigma T} / \epsilon$. Lemma 2 is proved.

Further, let us estimate the difference of solutions of the adjoint problems

$$
\Delta \psi(x, t ; F)=\psi(x, t ; F+\Delta F)-\psi(x, t ; F) .
$$


Lemma 3. The following estimate is valid with a constant $C_{1}$ :

$$
\|\Delta \psi(\cdot, \cdot ; F)\|_{L^{2}\left(\Omega_{T}\right)} \leq C_{1}\|\Delta F\|_{L^{2}\left(\Omega_{T}\right)} .
$$

Proof. Proof is similar to the proof of the previous lemma. Observing (3.7)(3.10) we see that the problem for $\Delta \psi(x, t ; F)$ has the following form:

$$
\begin{aligned}
& \Delta \psi_{t}(x, t ; F)=-A \Delta \psi(x, t ; F)+\int_{t}^{T} m(\tau-t) A \Delta \psi(x, \tau ; F) d \tau \quad \text { in } \Omega_{T}, \\
& \Delta \psi(x, T ; F)=2 \Delta u(x, T ; F) \text { in } \Omega, \\
& \Delta \psi(x, t ; F)=0 \text { in } \Gamma_{1, T} \text {, } \\
& -\nu_{A} \cdot \nabla \Delta \psi(x, t ; F)+\int_{t}^{T} m(\tau-t) \nu_{A} \cdot \nabla \Delta \psi(x, \tau ; F) d \tau \\
& =\vartheta \Delta \psi(x, t ; F) \text { in } \Gamma_{2, T} .
\end{aligned}
$$

We start by proving the assertion in case $\|m\|_{L^{1}(0, T)}$ is small enough and the equation (3.3) contains an additional term, namely it has the form

$$
\begin{aligned}
\Delta \psi_{t}(x, t ; F)= & -A \Delta \psi(x, t ; F)+\sigma \Delta \psi(x, t ; F) \\
& +\int_{t}^{T} m(\tau-t) A \Delta \psi(x, \tau ; F) d \tau \text { in } \Omega_{T},
\end{aligned}
$$

where $\sigma$ is again sufficiently large, i.e. $\sigma-a(x) \geq \epsilon$ for any $x \in \Omega$. Since $\Delta u \in W_{2}^{2,1}\left(\Omega_{T}\right)$, by the trace theorem it holds $\left.\Delta u\right|_{t=T} \in H^{1}(\Omega)$. Moreover, one can immediately check that the time-inverted function $\Delta \psi(x, T-t ; F)$ satisfies a problem of the form (2.1)-(2.4) with an homogeneous equation, homogeneous boundary conditions and the initial condition $2 \Delta u(x, T ; F)$. Therefore, applying Theorem 1 we see that the function $\Delta \psi(x, t ; F)$ belongs to $W_{2}^{2,1}\left(\Omega_{T}\right)$ and satisfies the problem (5.14), (5.11), (5.12), (5.13) in the classical sense. For the sake of shortness we omit the argument $F$ of $\Delta \psi$ and $\Delta u$ in forthcoming computations. Multiplying (5.14) by $\Delta \psi$ and integrating by parts we obtain

$$
\begin{aligned}
0= & \iint_{\Omega_{T}}\left[\Delta \psi_{t}+(A-\sigma) \Delta \psi-\int_{t}^{T} m(\tau-t) A \Delta \psi(x, \tau) d \tau\right] \Delta \psi d x d t \\
= & \frac{1}{2} \iint_{\Omega_{T}}\left[\Delta \psi^{2}\right]_{t} d x d t-\iint_{\Omega_{T}}\left[\sum_{i, j=1}^{n} a_{i j} \Delta \psi_{x_{j}} \Delta \psi_{x_{i}}+(\sigma-a) \Delta \psi^{2}\right] d x d t \\
& +\iint_{\Omega_{T}}\left[\sum_{i, j=1}^{n} a_{i j}(x) \int_{t}^{T} m(\tau-t) \Delta \psi_{x_{j}}(x, \tau) d \tau \Delta \psi_{x_{i}}(x, t)\right. \\
& \left.-a(x) \int_{t}^{T} m(\tau-t) \Delta \psi(x, \tau) d \tau \Delta \psi(x, t)\right] d x d t-\iint_{\Gamma_{2, T}} \vartheta \Delta \psi^{2} d \Gamma d t .
\end{aligned}
$$

Observing the final condition (5.11) and rearranging the terms we get

$$
\frac{1}{2} \int_{\Omega}[\Delta \psi(x, 0)]^{2} d x+\iint_{\Gamma_{2}, T} \vartheta \Delta \psi^{2} d \Gamma d t
$$




$$
\begin{aligned}
& +\iint_{\Omega_{T}}\left[\sum_{i, j=1}^{n} a_{i j} \Delta \psi_{x_{j}} \Delta \psi_{x_{i}}+(\sigma-a) \Delta \psi^{2}\right] d x d t \\
= & \iint_{\Omega_{T}}\left[\sum_{i, j=1}^{n} a_{i j}(x) \int_{t}^{T} m(\tau-t) \Delta \psi_{x_{j}}(x, \tau) d \tau \Delta \psi_{x_{i}}(x, t)\right. \\
- & \left.a(x) \int_{t}^{T} m(\tau-t) \Delta \psi(x, \tau) d \tau \Delta \psi(x, t)\right] d x d t+\frac{1}{2} \int_{\Omega}[\Delta u(x, T)]^{2} d x .
\end{aligned}
$$

The left-hand side of (5.15) is estimated from below:

$$
\begin{aligned}
& \frac{1}{2} \int_{\Omega}[\Delta \psi(x, 0)]^{2} d x+\iint_{\Gamma_{2, T}} \vartheta \Delta \psi^{2} d \Gamma d t+\iint_{\Omega_{T}}\left[\sum_{i, j=1}^{n} a_{i j} \Delta \psi_{x_{j}} \Delta \psi_{x_{i}}\right. \\
& \left.\quad+(\sigma-a) \Delta \psi^{2}\right] d x d t \geq \epsilon\left[\||\nabla \Delta \psi|\|_{L^{2}\left(\Omega_{T}\right)}^{2}+\|\Delta \psi\|_{L^{2}\left(\Omega_{T}\right)}^{2}\right]=: S^{2} .
\end{aligned}
$$

For the right-hand side of (5.15) we use the Cauchy-Schwarz inequality:

$$
\begin{aligned}
\iint_{\Omega_{T}} & {\left[\sum_{i, j=1}^{n} a_{i j}(x) \int_{t}^{T} m(\tau-t) \Delta \psi_{x_{j}}(x, \tau) d \tau \Delta \psi_{x_{i}}(x, t)\right.} \\
& \left.-a(x) \int_{t}^{T} m(\tau-t) \Delta \psi(x, \tau) d \tau \Delta \psi(x, t)\right] d x d t+\frac{1}{2} \int_{\Omega}[\Delta u(x, T)]^{2} d x \\
\leq & \hat{C}_{1}\left[\sum_{i, j=1}^{n}\left\|\int_{t}^{T} m(\tau-t) \Delta \psi_{x_{j}}(x, \tau) d \tau\right\|_{L^{2}\left(\Omega_{T}\right)}\left\|\Delta \psi_{x_{i}}\right\|_{L^{2}\left(\Omega_{T}\right)}\right. \\
+ & \left.\left\|\int_{t}^{T} m(\tau-t) \Delta \psi(x, \tau) d \tau\right\|_{L^{2}\left(\Omega_{T}\right)}\|\Delta \psi\|_{L^{2}\left(\Omega_{T}\right)}\right]+\frac{1}{2}\|\Delta u(\cdot, T)\|_{L^{2}(\Omega)}^{2}
\end{aligned}
$$

with some constant $\hat{C}_{1}$. It is easy to check by means of the change of variables of integration that

$$
\left\|\int_{t}^{T} m(\tau-t) v(x, \tau) d \tau\right\|_{L^{2}\left(\Omega_{T}\right)}=\|m * v\|_{L^{2}\left(\Omega_{T}\right)} \text { for any } v .
$$

Therefore, using the Young's inequality we get

$$
\begin{aligned}
& \left\|\int_{t}^{T} m(\tau-t) \Delta \psi_{x_{j}}(x, \tau) d \tau\right\|_{L^{2}\left(\Omega_{T}\right)} \leq\|m\|_{L^{1}(0, T)}\left\|\Delta \psi_{x_{j}}\right\|_{L^{2}\left(\Omega_{T}\right)}, \\
& \left\|\int_{t}^{T} m(\tau-t) \Delta \psi(x, \tau) d \tau\right\|_{L^{2}\left(\Omega_{T}\right)} \leq\|m\|_{L^{1}(0, T)}\|\Delta \psi\|_{L^{2}\left(\Omega_{T}\right)} .
\end{aligned}
$$

By means of (5.17)-(5.19) from (5.17) we obtain the relation

$$
\begin{aligned}
S^{2} \leq & \hat{C}_{1}\|m\|_{L^{1}(0, T)}\left[\sum_{i, j=1}^{n}\left\|\Delta \psi_{x_{j}}\right\|_{L^{2}\left(\Omega_{T}\right)}\left\|\Delta \psi_{x_{i}}\right\|_{L^{2}\left(\Omega_{T}\right)}+\|\Delta \psi\|_{L^{2}\left(\Omega_{T}\right)}^{2}\right] \\
& +\frac{1}{2}\|\Delta u(\cdot, T)\|_{L^{2}(\Omega)}^{2} .
\end{aligned}
$$


Like in the proof of Lemma 3 from this relation and the definition of $S$ we deduce the estimate $\|\Delta \psi\|_{L^{2}\left(\Omega_{T}\right)} \leq \frac{1}{\sqrt{\epsilon}}\|\Delta u(\cdot, T)\|_{L^{2}(\Omega)}$ provided $m$ satisfies the inequality

$$
\|m\|_{L^{1}(0, T)} \leq \frac{\epsilon}{2 \hat{C}_{1} n^{2}}
$$

Further, applying Lemma 2 to the obtained estimate we get (5.9) with the constant $C_{1}=C_{0} / \sqrt{\epsilon}$.

Finally, let us consider the original problem for $\Delta \psi$ without the additional $\sigma$-term and arbitrarily large $m$. Define $\Delta \psi_{\sigma}(x, t)=e^{-\sigma(T-t)} \Delta u(x, t)$ with $\sigma \in \mathbb{R}$. Then $\Delta \psi_{\sigma}$ solves the following problem:

$$
\begin{aligned}
& \Delta \psi_{\sigma, t}(x, t)=-A \Delta \psi_{\sigma}(x, t)+\int_{t}^{T} m_{\sigma}(\tau-t) A \Delta \psi_{\sigma}(x, \tau) d \tau \quad \text { in } \Omega_{T}, \\
& \Delta \psi_{\sigma}(x, T)=2 \Delta u(x, T) \quad \text { in } \Omega, \quad \Delta \psi_{\sigma}(x, t)=0 \quad \text { in } \Gamma_{1, T}, \\
& -\nu_{A} \cdot \nabla \Delta \psi_{\sigma}(x, t)+\int_{t}^{T} m_{\sigma}(\tau-t) \nu_{A} \cdot \nabla \Delta \psi_{\sigma}(x, \tau ;) d \tau=\vartheta \Delta \psi_{\sigma}(x, t) \quad \text { in } \Gamma_{2, T},
\end{aligned}
$$

where $m_{\sigma}(t)=e^{-\sigma t} m(t)$ again. There exists a sufficiently large $\sigma$ such that $m_{\sigma}$ satisfies the condition (5.20) and the inequality $\sigma-a(x) \geq \epsilon$ is valid for $x \in \Omega$. Thus, applying the first part of the proof to $\Delta \psi_{\sigma}$ we have

$$
\left\|\Delta \psi_{\sigma}\right\|_{L^{2}\left(\Omega_{T}\right)} \leq \frac{C_{0}}{\sqrt{\epsilon}}\|\Delta F\|_{L^{2}\left(\Omega_{T}\right)}
$$

Since $\left\|\Delta \psi_{\sigma}\right\|_{L^{2}\left(\Omega_{T}\right)} \geq e^{-\sigma T}\|\Delta \psi\|_{L^{2}\left(\Omega_{T}\right)}$ we reach the estimate (5.9) with the constant $C_{1}=C_{0} e^{\sigma T} / \sqrt{\epsilon}$. Lemma 3 is proved.

\section{Frechet Derivative and Gradient Method}

It follows from Lemma 2 with (3.2) that the functional $J$ is Frechet differentiable in $L^{2}\left(\Omega_{T}\right)$. Moreover, according to Lemma $1, J^{\prime}(F)$ is identical to the element $\psi(F)=\psi(x, t ; F)$ in $L^{2}\left(\Omega_{T}\right)$, i.e. it holds

$$
J^{\prime}(F) \tilde{F}=(\psi(F), \tilde{F})_{L^{2}\left(\Omega_{T}\right)}=\iint_{\Omega_{T}} \psi(x, t ; F) \tilde{F}(x, t) d x d t \quad \forall \tilde{F} \in L^{2}\left(\Omega_{T}\right) .
$$

Similarly, $J_{\alpha}$ is Frechet differentiable in $L^{2}\left(\Omega_{T}\right)$ and

$$
\begin{aligned}
J_{\alpha}^{\prime}(F) \tilde{F} & =(2 \alpha F+\psi(F), \tilde{F})_{L^{2}\left(\Omega_{T}\right)} \\
& =\iint_{\Omega_{T}}(2 \alpha F(x, t)+\psi(x, t ; F)) \tilde{F}(x, t) d x d t \quad \forall \tilde{F} \in L^{2}\left(\Omega_{T}\right) .
\end{aligned}
$$

Therefore, gradient-type methods can be used to solve the minimization problems (3.1) and (4.1). These methods must be combined by proper projection techniques to get minimum in the subset $\mathcal{F}$. However, it is possible to simplify 
the minimization procedure in case the structure of the subspace $\widehat{\mathcal{F}}$ is simple. In particular, global optimization can be used if $\mathcal{F}=\widehat{\mathcal{F}}$. To this end, let us consider the cases 1-3 introduced in Section 3.

Case 1. We introduce the functional $\Phi_{1, \alpha}(w)=J_{\alpha}(\varkappa w)$ with $\alpha \geq 0$ and the set $\mathcal{W}_{1}=\left\{w \in L^{2}(\Omega): \varkappa w \in \mathcal{F}\right\}$. Then the problem (4.1) (in case $\alpha=0$ the problem (3.1)) can be rewritten as follows:

$$
\text { find } w^{*}=\arg \min _{w \in \mathcal{W}_{1}} \Phi_{1, \alpha}(w) \text {. }
$$

In particular, when $\mathcal{F}=\widehat{\mathcal{F}}$, it holds $\mathcal{W}_{1}=L^{2}(\Omega)$ and we have a global minimization problem. Since $J_{\alpha}$ is Frechet differentiable, $\Phi_{1, \alpha}$ is also Frechet differentiable. Moreover, from (6.1) we deduce

$$
J_{\alpha}^{\prime}(\varkappa w) \varkappa \tilde{w}=\int_{\Omega}\left[\int_{0}^{T}[2 \alpha w(x) \varkappa(t)+\psi(x, t, \varkappa w)] \varkappa(t) d t\right] \tilde{w}(x) d x .
$$

This shows that $\Phi_{1, \alpha}^{\prime}(w)$ is identical to the element $\int_{0}^{T}[2 \alpha w(x) \varkappa(t)+$ $\psi(x, t, \varkappa w)] \varkappa(t) d t$ of $L^{2}(\Omega)$, that is

$$
\Phi_{1, \alpha}^{\prime}(w) \tilde{w}=\left(\int_{0}^{T}[2 \alpha w \varkappa(t)+\psi(\cdot, t, \varkappa w)] \varkappa(t) d t, \tilde{w}\right)_{L^{2}(\Omega)} \quad \forall \tilde{w} \in L^{2}(\Omega) .
$$

Using Cauchy-Schwarz inequality and Lemma 3 we estimate

$$
\begin{aligned}
& \left\|\Phi_{1, \alpha}^{\prime}(w+\Delta w)-\Phi_{1, \alpha}^{\prime}(w)\right\|_{L^{2}(\Omega)} \\
& =\left[\int_{\Omega}\left\{\int_{0}^{T}[2 \alpha \Delta w(x) \varkappa(t)+\psi(x, t, \varkappa(w+\Delta w))-\psi(x, t, \varkappa w)] \varkappa(t) d t\right\}^{2} d x\right]^{1 / 2} \\
& \leq\|2 \alpha \Delta w(x) \varkappa(t)+\psi(x, t, \varkappa(w+\Delta w))-\psi(x, t, \varkappa w)\|_{L^{2}\left(\Omega_{T}\right)}\|\varkappa\|_{L^{2}(0, T)} \\
& \leq\left(2 \alpha+C_{1}\right)\|\varkappa \Delta w\|_{L^{2}\left(\Omega_{T}\right)}\|\varkappa\|_{L^{2}(0, T)}=\left(2 \alpha+C_{1}\right)\|\varkappa\|_{L^{2}(0, T)}^{2}\|\Delta w\|_{L^{2}(\Omega)} .
\end{aligned}
$$

This implies that $\Phi_{1, \alpha}^{\prime}$ is uniformly Lipschitz-continuous, i.e.

$$
\left\|\Phi_{1, \alpha}^{\prime}(w+\Delta w)-\Phi_{1, \alpha}^{\prime}(w)\right\|_{L^{2}(\Omega)} \leq L_{\alpha}\|\Delta w\|_{L^{2}(\Omega)}
$$

where $L_{\alpha}=\left(2 \alpha+C_{1}\right)\|\varkappa\|_{L^{2}(0, T)}^{2}$.

The cases 2 and 3 can be treated in a similar manner. Let us summarize the results in these cases.

Case 2. Define $\Phi_{2, \alpha}(w)=J_{\alpha}(\varkappa w)$ with $\alpha \geq 0$ and the set $\mathcal{W}_{2}=\{w \in$ $\left.L^{2}\left(S_{T}\right): \varkappa w \in \mathcal{F}\right\}$. If $\mathcal{F}=\widehat{\mathcal{F}}$ then $\mathcal{W}_{2}=L^{2}\left(S_{T}\right)$. The problem (4.1) can be rewritten in the form: find $w^{*}=\arg \min _{w \in \mathcal{W}_{2}} \Phi_{2, \alpha}(w)$. The functional $\Phi_{2, \alpha}$ is Frechet differentiable, $\Phi_{2, \alpha}^{\prime}(w)$ is identical to the element $\int_{0}^{l}\left[2 \alpha w(\bar{x}, t) \varkappa\left(x_{n}\right)+\right.$ $\psi(x, t, \varkappa w)] \varkappa\left(x_{n}\right) d x_{n}$ of $L^{2}\left(S_{T}\right)$ and the uniform Lipschitz-estimate

$$
\left\|\Phi_{2, \alpha}^{\prime}(w+\Delta w)-\Phi_{2, \alpha}^{\prime}(w)\right\|_{L^{2}\left(S_{T}\right)} \leq L_{\alpha}\|\Delta w\|_{L^{2}\left(S_{T}\right)}
$$

is valid with $L_{\alpha}=\left(2 \alpha+C_{1}\right)\|\varkappa\|_{L^{2}(0, l)}^{2}$. 
Case 3. Let $\Phi_{3, \alpha}(w)=J_{\alpha}\left(\sum_{j=1}^{N} w_{j} \varkappa_{j}\right)$ with $\alpha \geq 0$ and $\mathcal{W}_{3}=\left\{w \in \mathbb{R}^{N}\right.$ : $\left.\sum_{j=1}^{N} w_{j} \varkappa_{j} \in \mathcal{F}\right\}$. If $\mathcal{F}=\widehat{\mathcal{F}}$ then $\mathcal{W}_{2}=\mathbb{R}^{N}$. The problem (4.1) admits the following form: find $w^{*}=\arg \min _{w \in \mathcal{W}_{3}} \Phi_{3, \alpha}(w)$. The functional $\Phi_{3, \alpha}$ is Frechet differentiable, $\Phi_{3, \alpha}^{\prime}(w)$ is identical to the element $\left(\iint_{\Omega_{T}}\left[2 \alpha \sum_{l=1}^{N} w_{l} \varkappa_{l}(x, t)+\right.\right.$ $\left.\left.\psi\left(x, t, \sum_{l=1}^{N} w_{l} \varkappa_{l}\right)\right] \varkappa_{j}(x, t) d x d t\right)_{j=1, \ldots, N}$ of $\mathbb{R}^{N}$ and the estimate

$$
\left\|\Phi_{3, \alpha}^{\prime}(w+\Delta w)-\Phi_{3, \alpha}^{\prime}(w)\right\|_{\mathbb{R}^{N}} \leq L_{\alpha}\|\Delta w\|_{\mathbb{R}^{N}}
$$

with $L_{\alpha}=\left(2 \alpha+C_{1}\right) \sum_{j=1}^{N}\left\|\varkappa_{j}\right\|_{L^{2}\left(\Omega_{T}\right)}^{2}$ is valid.

In the following, let $\Phi_{\alpha}$ be one of the functionals $\Phi_{j, \alpha}, j=1,2,3$, defined above and $\mathcal{W}$ be the corresponding set of admissible solutions $\mathcal{W}_{j}$. Then we consider the problem

$$
\text { find } w^{*}=\arg \min _{w \in \mathcal{W}} \Phi_{\alpha}(w) \text {. }
$$

For the sake of simplicity, we assume that $\mathcal{F}=\widehat{\mathcal{F}}$. This means that we consider the unconstrained minimization and $\mathcal{W}$ is $L^{2}(\Omega), L^{2}\left(S_{T}\right)$ and $\mathbb{R}^{N}$ in the cases 1, 2 and 3, respectively. Let $w_{0} \in \mathcal{W}$ be an initial guess and compute the successive approximations by means of the gradient method

$$
w_{k+1}=w_{k}-c_{k} \Phi_{\alpha}^{\prime}\left(w_{k}\right), \quad k=0,1,2, \ldots
$$

with steps $c_{k}>0$. Let us perform a little analysis for this iteration process following partially the example of [2].

Lemma 4. For any $\alpha \geq 0$ it holds

$$
\left|\Phi_{\alpha}\left(w_{k+1}\right)-\Phi_{\alpha}\left(w_{k}\right)-\Phi_{\alpha}^{\prime}\left(w_{k}\right)\left(w_{k+1}-w_{k}\right)\right| \leq \frac{L_{\alpha}}{2}\left\|w_{k+1}-w_{k}\right\|^{2} .
$$

Proof. Using the relation

$$
\Phi_{\alpha}\left(w_{k+1}\right)-\Phi_{\alpha}\left(w_{k}\right)=\int_{0}^{1} \Phi_{\alpha}^{\prime}\left(w_{k}+\tau\left(w_{k+1}-w_{k}\right)\right)\left(w_{k+1}-w_{k}\right) d \tau
$$

and the estimates $(6.3)-(6.5)$ we deduce

$$
\begin{aligned}
& \left|\Phi_{\alpha}\left(w_{k+1}\right)-\Phi_{\alpha}\left(w_{k}\right)-\Phi_{\alpha}^{\prime}\left(w_{k}\right)\left(w_{k+1}-w_{k}\right)\right| \\
& \quad=\left|\int_{0}^{1}\left[\Phi_{\alpha}^{\prime}\left(w_{k}+\tau\left(w_{k+1}-w_{k}\right)\right)-\Phi_{\alpha}^{\prime}\left(w_{k}\right)\right]\left(w_{k+1}-w_{k}\right) d \tau\right| \\
& \quad \leq L_{\alpha}\left\|w_{k+1}-w_{k}\right\|^{2} \int_{0}^{1} \tau d \tau=\frac{L_{\alpha}}{2}\left\|w_{k+1}-w_{k}\right\|^{2} .
\end{aligned}
$$

This proves (6.8).

Theorem 4. Let $\alpha \geq 0$ and $\delta \leq c_{k} \leq 2 / L_{\alpha}-\delta$ for any $k=0,1,2, \ldots$ where $\delta$ is some number in the half-interval $\left(0,1 / L_{\alpha}\right]$. Then the sequence $\Phi_{\alpha}\left(w_{k}\right)$ is 
monotonically decreasing, has a limit and the following relations are valid with $q_{k}=c_{k}-L_{\alpha} c_{k}^{2} / 2 \geq \delta-L_{\alpha} \delta^{2} / 2>0$ :

$$
\begin{aligned}
& \Phi_{\alpha}\left(w_{k}\right)-\Phi_{\alpha}\left(w_{k+1}\right) \geq q_{k}\left\|\Phi_{\alpha}^{\prime}\left(w_{k}\right)\right\|^{2}, \quad k=0,1,2, \ldots \\
& \Phi_{\alpha}^{\prime}\left(w_{k}\right) \rightarrow 0 \quad \text { as } k \rightarrow \infty \\
& \left\|w_{k+1}-w_{k}\right\|^{2} \leq \frac{c_{k}^{2}}{q_{k}}\left[\Phi_{\alpha}\left(w_{k}\right)-\Phi_{\alpha}\left(w_{k+1}\right)\right], \quad k=0,1,2, \ldots
\end{aligned}
$$

Proof. Due to (6.7) it hold $\left\|w_{k+1}-w_{k}\right\|^{2} \leq c_{k}^{2}\left\|\Phi_{\alpha}^{\prime}\left(w_{k}\right)\right\|^{2}$ and

$$
\Phi_{\alpha}^{\prime}\left(w_{k}\right)\left(w_{k+1}-w_{k}\right)=\left(\Phi_{\alpha}^{\prime}\left(w_{k}\right),-c_{k} \Phi_{\alpha}^{\prime}\left(w_{k}\right)\right)_{\mathcal{W}}=-c_{k}\left\|\Phi_{\alpha}^{\prime}\left(w_{k}\right)\right\|^{2} .
$$

Thus, by means of (6.8) we get

$$
\begin{aligned}
& \Phi_{\alpha}\left(w_{k+1}\right)-\Phi_{\alpha}\left(w_{k}\right)+c_{k}\left\|\Phi_{\alpha}^{\prime}\left(w_{k}\right)\right\|^{2} \\
& \quad \leq\left|\Phi_{\alpha}\left(w_{k+1}\right)-\Phi_{\alpha}\left(w_{k}\right)+c_{k}\left\|\Phi_{\alpha}^{\prime}\left(w_{k}\right)\right\|^{2}\right| \leq \frac{L_{\alpha} c_{k}^{2}}{2}\left\|\Phi_{\alpha}^{\prime}\left(w_{k}\right)\right\|^{2} .
\end{aligned}
$$

This yields $\Phi_{\alpha}\left(w_{k}\right)-\Phi_{\alpha}\left(w_{k+1}\right) \geq\left(c_{k}-\frac{L_{\alpha} c_{k}^{2}}{2}\right)\left\|\Phi_{\alpha}^{\prime}\left(w_{k}\right)\right\|^{2}$, i.e. (6.9). Due to $q_{k}>0$, the relation (6.9) implies that $\Phi_{\alpha}\left(w_{k}\right)$ is monotonically decreasing and since $\Phi_{\alpha}(w)$ has the lower bound 0 , the sequence $\Phi_{\alpha}\left(w_{k}\right)$ converges. Further, since the sequence $q_{k}$ has the positive lower bound $\delta-\frac{L_{\alpha} \delta^{2}}{2}$ and the left hand side of (6.9) converges to zero, we obtain (6.10). Finally, estimating (6.7) we have $\left\|w_{k+1}-w_{k}\right\|^{2}=c_{k}^{2}\left\|\Phi_{\alpha}^{\prime}\left(w_{k}\right)\right\|^{2}$. Using here (6.9) we obtain (6.11). Theorem is proved.

Clearly, the highest decrease rate of $\Phi_{\alpha}\left(w_{k}\right)$ is achieved in case $c_{k}=1 / L_{\alpha}$ when $q_{k}$ has the biggest value $q_{k}=1 / 2 L_{\alpha}$.

Theorem 5. Let $\alpha>0$ and $c_{k}$ be chosen as in Theorem 4 . Then the sequence $w_{k}$ strongly converges to the unique solution of the minimization problem (6.6).

Proof. The existence of the unique solution for the minimization problem immediately follows from Theorem 3 and the definitions of $\Phi_{\alpha}$. Moreover, since $J_{\alpha}$ is weakly sequentially lower semi-continuous, strictly convex and weakly coercive (see the proof of Theorem 3), the same properties are valid also for $\Phi_{\alpha}$. It is well-known that under such properties every minimizing sequence of $\Phi_{\alpha}$ weakly converges to the minimum point $w^{*}$. Thus, firstly, let us show that $w_{k}$ is a minimizing sequence, i.e. $\Phi_{\alpha}\left(w_{k}\right) \rightarrow \Phi_{\alpha}\left(w^{*}\right)$.

Note that the sequence $w_{k}$ is bounded. Indeed, otherwise there exists a subsequence $w_{k_{i}}$ such that $\left\|w_{k_{i}}\right\| \rightarrow \infty$ and by the weak coercitivity it holds $\Phi_{\alpha}\left(w_{k_{i}}\right) \rightarrow \infty$ which contradicts to the statement of Theorem 4 that $\Phi_{\alpha}\left(w_{k}\right)$ is monotonically decreasing.

Since $\Phi_{\alpha}$ is convex, its Frechet derivative is monotone, i.e.

$$
\left[\Phi_{\alpha}^{\prime}(\widetilde{w})-\Phi_{\alpha}^{\prime}(w)\right](\widetilde{w}-w) \geq 0 \quad \forall w, \widetilde{w} \in \mathcal{W}
$$


Let us choose some $\tau \in(0,1)$. Observing that it holds $\Phi_{\alpha}^{\prime}\left(w^{*}\right)=0$ in the global minimum point $w^{*}$ and applying (6.12) with $w=w^{*}$ and $\widetilde{w}=w^{*}+\tau\left(w_{k}-w^{*}\right)$ we have

$$
\begin{gathered}
\liminf _{k \rightarrow \infty} \Phi_{\alpha}^{\prime}\left(w^{*}+\tau\left(w_{k}-w^{*}\right)\right)\left(w_{k}-w^{*}\right)=\frac{1}{\tau} \liminf _{k \rightarrow \infty}\left[\Phi_{\alpha}^{\prime}\left(w^{*}+\tau\left(w_{k}-w^{*}\right)\right)\right. \\
\left.-\Phi_{\alpha}^{\prime}\left(w^{*}\right)\right]\left(w^{*}+\tau\left(w_{k}-w^{*}\right)-w^{*}\right) \geq 0 .
\end{gathered}
$$

On the other hand, it holds $\lim _{k \rightarrow \infty} \Phi_{\alpha}^{\prime}\left(w_{k}\right)\left(w_{k}-w^{*}\right)=0$ because of the boundedness of $w_{k}$ and the relation (6.10). Thus, using (6.12) with $w=w_{k}$ and $\widetilde{w}=w^{*}+\tau\left(w_{k}-w^{*}\right)$ we obtain

$$
\begin{gathered}
\limsup _{k \rightarrow \infty} \Phi_{\alpha}^{\prime}\left(w^{*}+\tau\left(w_{k}-w^{*}\right)\right)\left(w_{k}-w^{*}\right)=\frac{1}{1-\tau} \limsup _{k \rightarrow \infty}\left[\Phi_{\alpha}^{\prime}\left(w^{*}+\tau\left(w_{k}-w^{*}\right)\right)\right. \\
\left.-\Phi_{\alpha}^{\prime}\left(w_{k}\right)\right]\left(w_{k}-w^{*}-\tau\left(w_{k}-w^{*}\right)\right) \leq 0
\end{gathered}
$$

The estimates (6.13) and (6.14) imply $\lim _{\sup _{k \rightarrow \infty}} v_{k} \leq 0 \leq \liminf _{k \rightarrow \infty} v_{k}$ for the sequence $v_{k}=\Phi_{\alpha}^{\prime}\left(w^{*}+\tau\left(w_{k}-w^{*}\right)\right)\left(w_{k}-w^{*}\right)$. Hence,

$$
\lim _{k \rightarrow \infty} \Phi_{\alpha}^{\prime}\left(w^{*}+\tau\left(w_{k}-w^{*}\right)\right)\left(w_{k}-w^{*}\right)=0 .
$$

Further, writing

$$
\Phi_{\alpha}\left(w_{k}\right)-\Phi_{\alpha}\left(w^{*}\right)=\int_{0}^{1} \Phi_{\alpha}^{\prime}\left(w^{*}+\tau\left(w_{k}-w^{*}\right)\right)\left(w_{k}-w^{*}\right) d \tau
$$

and using (6.15) we obtain $\Phi_{\alpha}\left(w_{k}\right)-\Phi_{\alpha}\left(w^{*}\right) \rightarrow 0$. This shows that $w_{k}$ is a minimizing sequence. Consequently, $w_{k} \rightarrow w^{*}$.

Now let us prove the assertion of the Theorem $w_{k} \rightarrow w^{*}$. In case 3 this is evident, because $\mathcal{W}$ is of finite dimension. Thus, let us study the cases 1 and 2. Then it holds $\Phi_{\alpha}(w)=\alpha \nu\|w\|^{2}+\Phi_{0}(w)$ where $\nu$ is a positive constant $\left(\nu=\int_{0}^{T} \varkappa^{2}(t) d t\right.$ in case 1 and $\nu=\int_{0}^{l} \varkappa^{2}\left(x_{n}\right) d x_{n}$ in case 2$)$. Since the norm is weakly lower sequentially semicontinuous, the relation $w_{k} \rightarrow w^{*}$ implies

$$
\left\|w^{*}\right\|^{2} \leq \liminf _{k \rightarrow \infty}\left\|w_{k}\right\|^{2} .
$$

On the other hand, since $\Phi_{\alpha}\left(w_{k}\right)$ converges to $\Phi_{\alpha}\left(w^{*}\right)$ and $\Phi_{0}(w)$ is weakly lower sequentially semicontinuous and we obtain

$$
\begin{aligned}
\limsup _{k \rightarrow \infty}\left\|w_{k}\right\|^{2}= & \frac{1}{\alpha \nu} \limsup _{k \rightarrow \infty}\left[\Phi_{\alpha}\left(w_{k}\right)-\Phi_{0}\left(w_{k}\right)\right] \\
& =\frac{1}{\alpha \nu}\left\{\lim _{k \rightarrow \infty} \Phi_{\alpha}\left(w_{k}\right)+\limsup _{k \rightarrow \infty}\left[-\Phi_{0}\left(w_{k}\right)\right]\right\} \\
& =\frac{1}{\alpha \nu}\left\{\Phi_{\alpha}\left(w^{*}\right)-\liminf _{k \rightarrow \infty} \Phi_{0}\left(w_{k}\right)\right\} \\
& \leq \frac{1}{\alpha \nu}\left\{\Phi_{\alpha}\left(w^{*}\right)-\Phi_{0}\left(w^{*}\right)\right\}=\left\|w^{*}\right\|^{2} .
\end{aligned}
$$

Putting together (6.16) and (6.17) we get $\limsup _{k \rightarrow \infty}\left\|w_{k}\right\|^{2} \leq\left\|w^{*}\right\|^{2}$ $\leq \liminf _{k \rightarrow \infty}\left\|w_{k}\right\|^{2}$. This gives $\lim _{k \rightarrow \infty}\left\|w_{k}\right\|^{2}=\left\|w^{*}\right\|^{2}$. Since in an Hilbert space the weak convergence and the convergence of norms implies the strong convergence, we prove $w_{k} \rightarrow w^{*}$. The proof is complete. 


\section{References}

[1] A.T. Ayapbergenova, S.I. Kabanikhin and A. Lorenzi. Justification of optimization methods for inverse integro-differential hyperbolic problems. J. Inverse Ill-Posed Probl., 12(5):457-479, 2004. Doi:10.1515/1569394042531341.

[2] A. Hasanov. Simultaneous determination of source terms in a linear parabolic problem from the final overdetermination: Weak solution approach. J. Math. Anal. Appl., 330(2):766-779, 2007. Doi:10.1016/j.jmaa.2006.08.018.

[3] V. Isakov. Inverse parabolic problems with the final overdetermination. Comm. Pure Appl. Math., 44(2):185-209, 1991. Doi:10.1002/cpa.3160440203.

[4] J. Janno. Determination of a time- and space-dependent heat flux relaxation function by means of a restricted Dirichlet-to-Neumann operator. Math. Meth. Appl. Sci., 27(11):1241-1260, 2004. Doi:10.1002/mma.490.

[5] J. Janno and K. Kasemets. A positivity principle for parabolic integro-differential equations and inverse problems with final overdetermination. Inverse Probl. Imaging, 3(1):17-41, 2009. Doi:10.3934/ipi.2009.3.17.

[6] J. Janno and A. Lorenzi. Recovering memory kernels in parabolic transmission problems. J. Inverse Ill-Posed Probl., 16(3):239-266, 2008. Doi:10.1515/JIIP.2008.015.

[7] J. Janno and L.v. Wolfersdorf. Inverse problems for memory kernels by laplace transform methods. Z. Anal. Anwend., 19(2):489-510, 2000.

[8] J. Janno and L.v. Wolfersdorf. Identification of memory kernels in onedimensional heat flow with boundary conditions of the third kind. Inverse Probl. Eng., 9(2):179-198, 2001. Doi:10.1080/174159701088027760.

[9] S.I. Kabanikhin and A. Lorenzi. Identification Problems of Wave Phenomena. Theory and Numerics. VSP, Utrecht, 1999.

[10] O.A. Ladyzhenskaya, V.A. Solonnikov and N.N. Ural'tseva. Linear and Quasilinear Equations of Parabolic Type. American Mathematical Society, Providence, RI, 1968.

[11] A. Lorenzi. An identification problem for a conserved phase-field model with memory. Math. Meth. Appl. Sci., 28(11):1315-1339, 2005. Doi:10.1002/mma.614.

[12] A. Lorenzi and G. Mola. Identification of unknown terms in convolution integrodifferential equations in a Banach space. J. Inverse Ill-Posed Probl., 18(3):321355, 2010. Doi:10.1515/JIIP.2010.013.

[13] E. Pais. Identification of memory kernels in heat flow measuring heat flux at the ends of the bar. Math. Model. Anal., 15(4):473-490, 2010. Doi:10.3846/1392-6292.2010.15.473-490.

[14] E. Pais and J. Janno. Inverse problem to determine degenerate memory kernels in heat flux with third kind boundary conditions. Math. Model. Anal., 11(4):427450, 2006. Doi:10.1080/13926292.2006.9637329.

[15] V.A. Solonnikov. On Boundary Value Problems for General Parabolic Systems of Differential Equations. Proc. Steklov Inst. Math., 83, Moscow, 1968. (in Russian)

[16] E. Zeidler. Applied Functional Analysis. Main Principles and Their Applications. Springer, New York, 1995. 